\title{
A study of resolution function on a MIEZE spectrometer
}

AUTHOR(S):

Hayashida, H; Hino, M; Kitaguchi, M; Kawabata, Y; Achiwa, N

\section{CITATION:}

Hayashida, H ... [et al]. A study of resolution function on a MIEZE spectrometer. Measurement Science and Technology 2008, 19(3): 034006.

ISSUE DATE:

2008-03-01

URL:

http://hdl.handle.net/2433/98013

\section{RIGHT:}

(C) 2008 IOP Publishing; This is not the published version. Please cite only the published version.; この論文は出版社版でありません。引用の 際には出版社版をご確認ご利用ください。 


\title{
A study of resolution function on MIEZE spectrometer
}

\author{
H Hayashida ${ }^{1}, \mathrm{M} \mathrm{Hino}^{2}, \mathrm{M} \mathrm{Kitaguchi}^{2}, \mathrm{Y} \mathrm{Kawabata}^{2}$ and N \\ Achiwa $^{3}$ \\ ${ }^{1}$ Faculty of Engineering, Kyoto University, Sakyo-ku, Kyoto 606-8501, Japan \\ ${ }^{2}$ Research Reactor Institute, Kyoto University, Kumatori, Osaka 590-0494, Japan \\ ${ }^{3}$ Graduate School of Science, Osaka University, Toyonaka,Osaka 560-0043, Japan \\ E-mail: hayashid@post3.rri.kyoto-u.ac.jp
}

\begin{abstract}
.
We have installed MIEZE (Modulated IntEnsity by Zero Effort) spectrometer to the C3-1-2-2(MINE1) port at JRR-3M. In the MIEZE spectrometer a sample is placed after the analyzer. Thus the contrast of MIEZE signal can be observed without reduction even in the case of magnetic scattering where the signal for NSE (Neutron Spin Echo) and NRSE (Neutron Resonance Spin Echo) instruments would be less than half. MIEZE signal is sensitive for dispersion of neutron trajectories from sample to detector. In other words MIEZE signal is reduced due to the dispersion alone even without in- and quasi-elastic scattering. We studied the correlation between experimental configuration and its resolution function using Monte Carlo simulation. We prepared a magnetite ferrofluid sample with a typical particle diameter of $84 \AA$, and examined the simulation model by experiments.
\end{abstract}

PACS numbers: 03.75.Be, 03.75.Dg, 61.12.Ex

Keywords: MIEZE, resolution function, ferrofluid

Submitted to: Meas. Sci. Technol. 


\section{Introduction}

MIEZE (Modulated IntEnsity by Zero Effort) is a variant of a Neutron Resonance Spin Echo (NRSE) spectrometer [1,2]. We have developed a MIEZE spectrometer at C3-1-22(MINE1) port JRR-3M in order to install it to the high flux pulsed neutron source at JPARC [3] in the near future. We succeeded in observing the MIEZE signal with effective frequency of $1 \mathrm{MHz}[4,5]$. In the MIEZE spectrometer the sample is placed after the analyzer and there are no optical components between sample and detector. Therefore the MIEZE technique can be applied to SANS (Small Angle Neutron Scattering) [6] and neutron reflectometry [7]. When we measure Neutron Spin Echo (NSE) and NRSE signals on paramagnetic and ferromagnetic samples the maximum contrast is reduced to 0.5 or less [8]. On the other hand the contrast of a MIEZE signal can be observed with almost $100 \%$ contrast(1) even in magnetic scattering [9] because the sample is placed after the analyzer. The energy resolution of a MIEZE spectrometer is a function of the so called Fourier time like for NSE and NRSE spectrometers. The MIEZE-Fourier time $\tau$ is described as, $\tau=\frac{2 \hbar \omega_{s} L_{s d}}{m v^{3}}$, where $\omega_{s}$ is the frequency of oscillating field in RSF (Resonance Spin Flipper), $L_{s d}$ is the path length between sample and detector, $v$ is the neutron velocity, $m$ is the mass of neutron, $\hbar$ is the reduced Planck's constant. The Fourier time is proportional to $\omega_{s}$ and $L_{s d}$. MIEZE signal becomes more sensitive for dispersion of $L_{s d}$ with higher frequencies $\omega_{s}$. When we consider the instrumental resolution function for NSE and NRSE at high resolution, the main object is to estimate the inhomogeneity of the precession fields. In case of MIEZE we have to consider the dispersion of path length $L_{s d}$ with sample. The dispersion depends on experimental arrangements. Indeed, the dispersion effect is negligible for most measurements when we use reflectometry geometry [7]. When we use a transmission geometry like a SANS, the effect is not small. We calculated the resolution function for transmission geometry by using Monte Carlo (MC) simulation and prepared magnetite ferrofluid as a sample.

We aim to investigate dynamical structure-changes of the magnetite ferrofluid by using the MIEZE spectrometer. The ferrofluid consists of magnetite particles and solvent (heavy water). The magnetite particles with a diameter in the order of $100 \AA$ are coated by oleic acid. We expect that there are two kind of dynamic modes in ferrofluid. One is diffusion of magnetite particles and the other is relaxation of superparamagnetism. The both dynamic modes depend on the magnetite particle size. The relaxation of the particle diffusion with the diameter $100 \AA$ is reported to be over 2 ns [10] but that of superparamagnetism is estimated to be the order of $0.1 \mathrm{~ns}$ with a particle diameter of $85 \AA$ at room temperature. In this experiment the Fourier time is restricted from 0.3 to $1.6 \mathrm{~ns}$. Thus we prepared ferrofluid sample in which particle size as small as possible by using a centrifugal separator. In this paper main purpose is to examine the MIEZE instrumental resolution function by comparing experimental results. 


\section{MC simulation of MIEZE signal}

\subsection{Simulation method}

A top view and energy diagram of neutron spin states of the MIEZE spectrometer which was installed in C3-1-2-2, MINE1 port is shown in figure 1. A MIEZE signal is observed
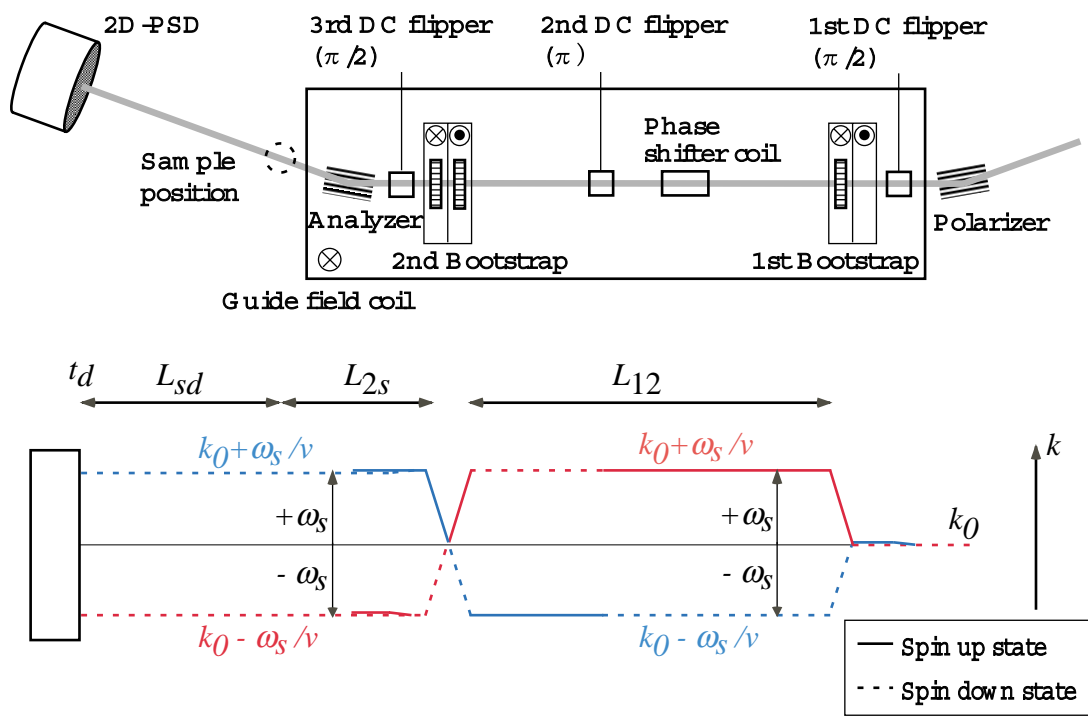

Figure 1. The schematic top view and energy level diagram of the MIEZE spectrometer which is installed in C3-1-2-2, MINE1 port at JRR-3M. The 2D-PSD (two-dimensional positionsensitive detector) consists of ${ }^{6} \mathrm{Li}$-enriched glass scintillator and a position sensitive photomultiplier of $5 "$ in diameter [11].

as a function of the phase difference between two energy states. The net phase difference between two energy states at the detector is given by

$$
\Phi=2 \omega_{s} t_{d}+\frac{2 \omega_{s}}{v}\left(-L_{12}+L_{2 s}+L_{s d}+\delta L_{s d}\right)
$$

where $t_{d}$ is the time at detector, $L_{12}$ is the length of the flight paths between first and second bootstrap coil, $L_{2 s}$ between second bootstrap, coil and sample position and $L_{s d}$ between sample position and detector, respectively. The $\delta L_{s d}$ is path length dispersion between sample and detector which appeared from scattering by sample. When a spinecho condition on MIEZE which is written as $L_{12}=L_{2 s}+L_{s d}$ is fulfilled, the net phase is given by

$$
\Phi=2 \omega_{s}\left(t_{d}+\frac{\delta L_{s d}}{v}\right)
$$

Neutron intensity $I_{d}$ is modulated as a function of $t_{d}$,

$$
I_{d} \propto \frac{1+\cos \Phi}{2} .
$$

The contrast $C$ which is observed on MIEZE spectrometer is given by

$$
C_{M I E Z E} \propto \frac{\int S(Q, \omega) I_{d}}{\int S(Q, \omega)} d \omega \propto \frac{\int S(Q, \omega) \cos \Phi}{\int S(Q, \omega)} d \omega
$$


where $\omega$ is the energy transfer of neutrons caused by quasielastic scattering. A schematic diagram of a $\delta L_{s d}$ calculation is shown in figure 2. The sample size in horizontal, vertical

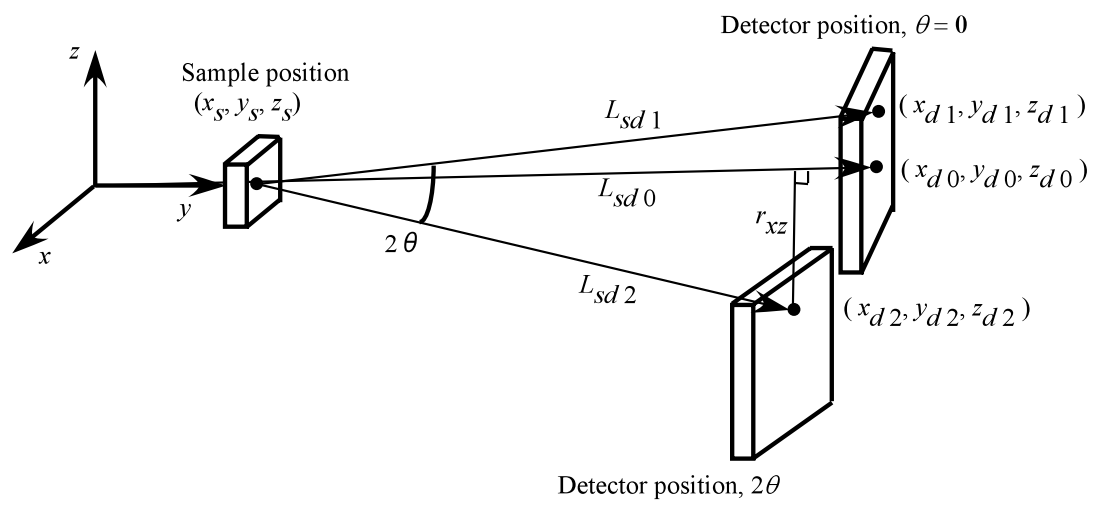

Figure 2. The Schematic diagram for $\delta L_{s d}$ calculation.

dimensions and sample thickness are defined as $x_{s}, z_{s}$ and $y_{s}$, respectively. Detector size in horizontal, vertical dimensions and effective thickness are defined as $x_{d}, z_{d}$ and $y_{d}$ respectively. Beam divergence in horizontal and vertical direction are defined as $\delta \theta_{x}$ and $\delta \theta_{z}$, respectively.

$$
\begin{aligned}
& x_{d}=L_{s d 0} \delta \theta_{x}+x_{s} \\
& x_{d 0}=\left\{\begin{array}{lll}
x_{d 0} & \text { for } x_{d 0}<x_{d} \\
x_{d} & \text { for } x_{d 0} \geq x_{d}
\end{array}\right. \\
& z_{d}=L_{s d} \delta \theta_{z}+z_{s} \\
& z_{d 0}=\left\{\begin{array}{lll}
z_{d 0} & \text { for } z_{d 0}<z_{d} \\
z_{d} & \text { for } z_{d 0} \geq z_{d}
\end{array}\right.
\end{aligned}
$$

$L_{s d 0}$ is the path lengths for incident neutrons without scattering by the sample. The path lengths for neutrons scattered by the sample are $L_{s d 1}$ and $L_{s d 2}$. The $L_{s d 1}$ is for scattering angle $\theta=0$ and the $L_{s d 2}$ is for $\theta \neq 0$. The sample position $\left(x_{s}, y_{s}, z_{s}\right)$ and detector positions $\left(x_{d 0}, y_{d 0}, z_{d 0}\right)$ and $\left(x_{d 1}, y_{d 1}, z_{d 1}\right)$ are given by random numbers, respectively. When the scattering angle of $\theta \neq 0$, detector is at the direction of $2 \theta$ and the coordinates $\left(x_{d 2}, y_{d 2}, z_{d 2}\right)$ on detector are calculated by using rotation matrix as follows,

$$
\left(\begin{array}{l}
x_{d 2} \\
y_{d 2}
\end{array}\right)=\left(\begin{array}{cc}
\cos 2 \theta & -\sin 2 \theta \\
\sin 2 \theta & \cos 2 \theta
\end{array}\right)\left(\begin{array}{l}
x_{d 1} \\
y_{d 1}
\end{array}\right), \quad z_{d 2}=z_{d 1} .
$$

Then $\delta L_{s d}$ is calculated by

$$
\begin{aligned}
& L_{s d i}=\sqrt{\left(x_{d i}-x_{s}\right)^{2}+\left(y_{d i}-y_{s}\right)^{2}+\left(z_{d i}-z_{s}\right)^{2}}, \quad i=0,1,2 \\
& \delta L_{s d}=L_{s d 2}-L_{s d 0} .
\end{aligned}
$$


Table 1. Parameters for figure 3. The variable numbers are $x_{s}$ and $y_{s}$.

\begin{tabular}{ll}
\hline sample slit and thickness & $\left(x_{s}, y_{s}, z_{s}\right)=\left(x_{s}, y_{s}, 20\right)$ \\
\hline detector slit and effective thickness & $\left(x_{d}, y_{d}, z_{d}\right)=(15,0.1,15)$ \\
\hline
\end{tabular}

Table 2. Parameters for figure 4 . The variable numbers are $z_{s}$ and $z_{d}$.

\begin{tabular}{ll}
\hline sample slit and thickness & $\left(x_{s}, y_{s}, z_{s}\right)=\left(2,2, z_{s}\right)$ \\
\hline detector slit and effective thickness & $\left(x_{d}, y_{d}, z_{d}\right)=\left(15,0.1, z_{d}\right)$ \\
\hline
\end{tabular}

When we think about only elastic scattering, $C_{M I E Z E}(t=0)$ is given by

$$
\begin{aligned}
& C_{M I E Z E}(t=0) \propto \int S(Q) \cos \Phi d V \\
& \simeq \sum_{i=1}^{N} S\left(Q_{i}\right) \cos \Phi_{i} d V=\sum_{i=1}^{N} S\left(Q_{i}\right) \cos \left(\frac{2 \omega_{s}}{v_{i}} \delta L_{s d(i)}\right) d V,
\end{aligned}
$$

where $i$ and $N$ are each and total number of neutron detected in the detector volume $d V$, respectively. We can observe $C_{M I E Z E}(t=0)=1$ in case of no path dispersion $\operatorname{effect}\left(\sum_{i=1}^{N} \delta L s d(i)=0\right)$.

\subsection{Simulation of resolution function}

The Eq.(13) shows that the phase shift term is proportional to $\omega_{s}$. In present the maximum oscillating frequency $\omega_{s}$ of the RSF on MIEZE is $0.5 \mathrm{MHz}$. However, the RSFs can work at $0.3 \mathrm{MHz}$ with great stability. Therefore, we calculate the $C_{M I E Z E}(t=0)$ with $\omega_{s}=0.3 \mathrm{MHz}$. At first $S(Q)$ is constant as a simple case. Here neutron wavelength $\lambda$ is $8.1 \AA$, wavelength bandwidth is $17.4 \%$ in full width half maximum and effective thickness of detector is $y_{d}=0.1 \mathrm{~mm}$. In the MIEZE at MINE1 port it is expected that $C_{M I E Z E}(t=0)$ is more sensitive to $x_{s}$ than $z_{s}$ since detector angle moves horizontally. $z_{s}$ is set to $20 \mathrm{~mm}$ at MINE1 port for high neutron intensity. In the MC simulation, $z_{s}$ is set to $20 \mathrm{~mm}$ as well. Both of $x_{d}$ and $z_{d}$ are set to $15 \mathrm{~mm}$. We study the path dispersion effect to $C_{M I E Z E}(t=0)$ caused by sample slit size and sample thickness by changing $x_{s}$ and $y_{s}$. Parameters are shown in table 1 , and the result is shown in figure 3 . This result shows that $C_{M I E Z E}(t=0)$ is very sensitive to $x_{s}$ and not so sensitive to $y_{s}$ in this geometry. The $C_{M I E Z E}(t=0)$ for $S(Q)=1$ decreases monotonically as a function of $Q$.

$S(Q)$ is given by core shell model because structure of magnetite ferrofluid can be understood by core shell model, which is described in detail in the next section. A simulation about the effect of $z_{s}$ and $z_{d}$ is carried out. Simulation parameters are shown in table 2 . Both of results shown in figure 4 are calculated with same beam divergence; becoming $z_{s}+z_{d}=$ constant. This result shows that the $C_{M I E Z E}(t=0)$ is more sensitive to $z_{d}$ than $z_{s}$. 


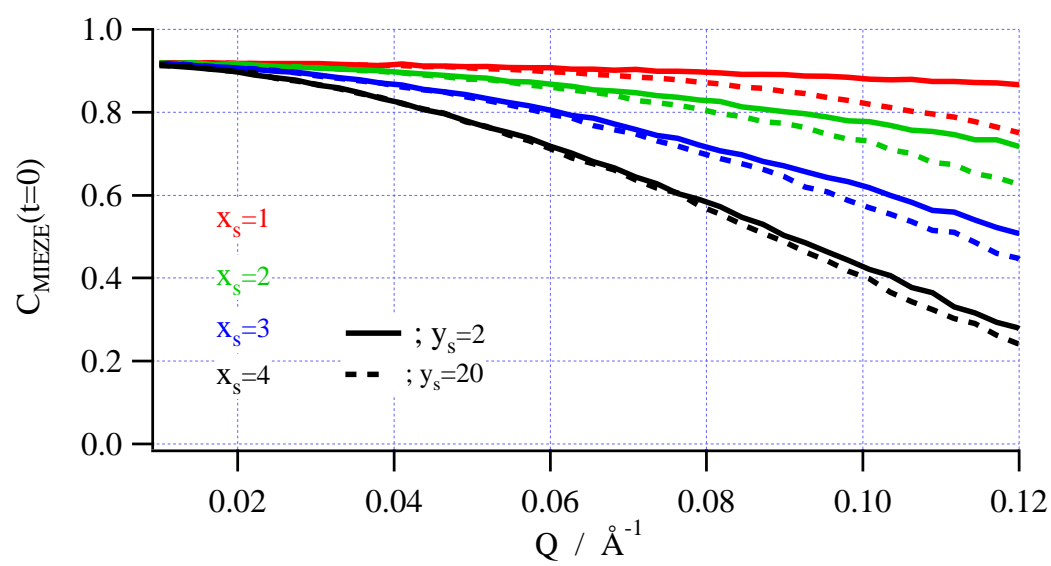

Figure 3. Simulation result of $C_{M I E Z E}(t=0)$ by changing the $x_{s}$ and $y_{s}$. Both of $x_{d}$ and $z_{d}$ are $15 \mathrm{~mm}$. The $x_{s}=1 \mathrm{~mm}, x_{s}=2 \mathrm{~mm}, x_{s}=3 \mathrm{~mm}$ and $x_{s}=4 \mathrm{~mm}$ are shown in red, green, blue and black, respectively. S(Q):constant.

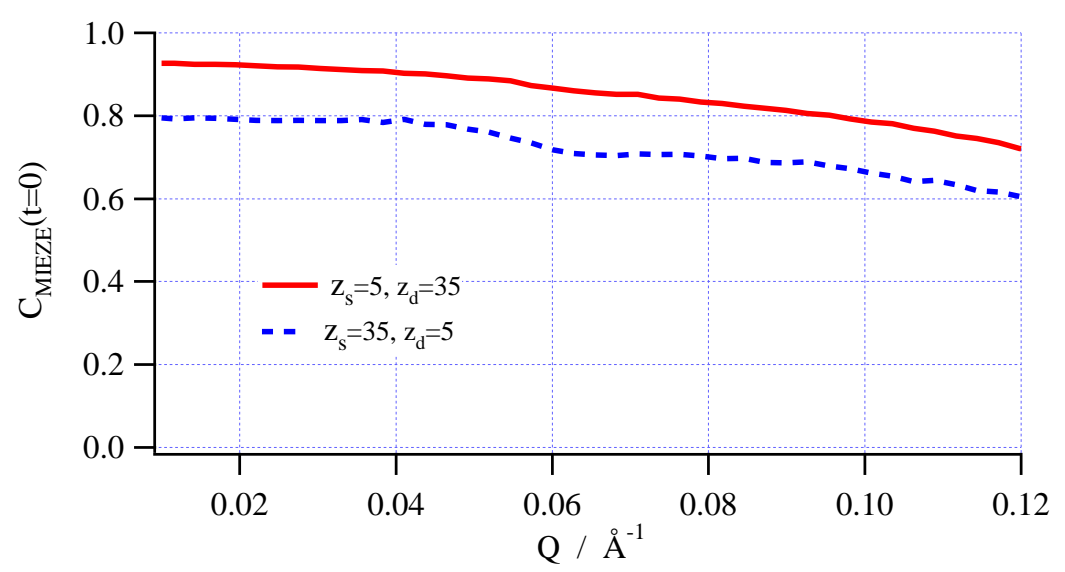

Figure 4. Simulation result of $C_{M I E Z E}(t=0)$ with the variable numbers of $z_{s}$ and $z_{d}$. Line is $z_{s}=35 \mathrm{~mm}$ and $z_{d}=5 \mathrm{~mm}$. Dashed line is $z_{s}=5 \mathrm{~mm}$ and $z_{d}=35 \mathrm{~mm}$. $\mathrm{S}(\mathrm{Q})$ :core shell model(see table 4 and 5$)$.

Table 3. Parameters for figure 5 . The variable number is $z_{d}$.

\begin{tabular}{ll}
\hline sample slit and thickness & $\left(x_{s}, y_{s}, z_{s}\right)=(2,2,20)$ \\
\hline detector slit and effective thickness & $\left(x_{d}, y_{d}, z_{d}\right)=\left(15,0.1, z_{d}\right)$ \\
\hline
\end{tabular}

We study about the effect of $z_{d}$ with constant of $z_{s}=20 \mathrm{~mm}$. The $z_{d}$ is changed from $5 \mathrm{~mm}$ to $40 \mathrm{~mm}$ in steps of $5 \mathrm{~mm}$. Other parameters in this simulation are shown in table 3. This result shows that $C_{M I E Z E}(t=0)$ is not sensitive to $z_{d}$ up to $10 \mathrm{~mm}$ as shown in figure 5 . In the $Q$ range of $0.06 \AA^{-1}, C_{M I E Z E}(t=0)$ changes drastically with $z_{d} \geq 30 \mathrm{~mm}$. This is expected that the $S(Q)$ which is given by core shell model affect to the $C_{M I E Z E}(t=0)$ with wide $z_{d}$. The $S(Q)$ is not so affect to $C_{M I E Z E}(t=0)$ with 


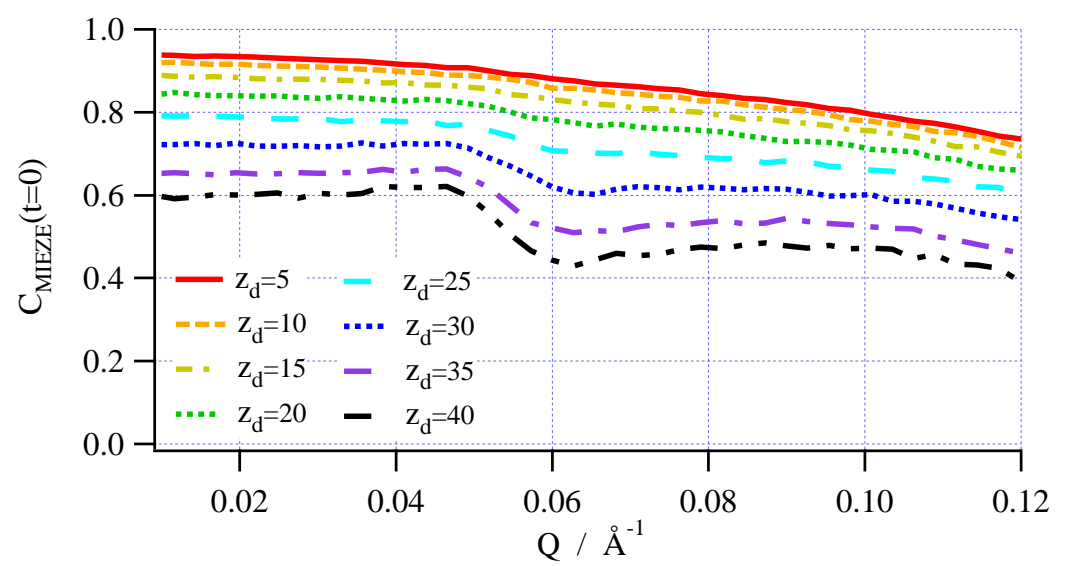

Figure 5. Simulation result of $C_{M I E Z E}(t=0)$ with a parameter of $z_{d}$. The $z_{d}$ is changed from $5 \mathrm{~mm}$ to $40 \mathrm{~mm}$. $\mathrm{S}(\mathrm{Q})$ :core shell model(see Table 4 and 5 ).

Table 4. Nuclear SLD of magnetite ferrofluid.

\begin{tabular}{ll}
\hline Core SLD & $\rho_{c}=6.97 \times 10^{-10} \mathrm{~cm}^{-2}$ \\
\hline Shell SLD & $\rho_{s}=-0.30 \times 10^{-10} \mathrm{~cm}^{-2}$ \\
\hline Solvent SLD & $\rho_{\text {solv }}=5.82 \times 10^{-10} \mathrm{~cm}^{-2}$ \\
\hline
\end{tabular}

$z_{d}=25$ and $20 \mathrm{~mm}$. In the $z_{d} \leq 15 \mathrm{~mm}$, the effect of $S(Q)$ disappear.

\section{Experimental results and discussion}

\subsection{Measurement of small angle scattering}

We measured small angle scattering by using Neutron Refrectometry (NR) at JRR-3M, C3-1-2-3, MINE2 port in order to determine the particle size. The concentration of magnetite was $5.86 \mathrm{wt} \%$ and solvent was $\mathrm{D}_{2} \mathrm{O}$. The neutron wavelength is $8.8 \AA$ and wavelength bandwidth is $2.7 \%$ in full width half maximum. The measurement result of small angle scattering was analyzed by using core shell model. Form factor of core shell model is given by

$$
\begin{aligned}
& S(Q) \propto \frac{1}{V_{s}}\left[\frac{3 V_{c}\left(\rho_{c}-\rho_{s}\right) j\left(Q r_{c}\right)}{Q r_{c}}+\frac{3 V_{s}\left(\rho_{s}-\rho_{\text {solv }}\right) j\left(Q r_{s}\right)}{Q r_{s}}\right]^{2}+b k g, \\
& j(x)=\frac{\sin x-x \cos x}{x^{2}}, \quad r_{s}=r_{c}+t \text { and } V_{i}=\frac{4 \pi r_{i}^{3}}{3},
\end{aligned}
$$

where the $r_{c}, V_{c}$ and $\rho_{c}$ are the radius, volume and scattering length density (SLD) of core, respectively. The $t, V_{s}$ and $\rho_{s}$ are the thickness, volume and SLD of shell, respectively. The $\rho_{\text {solv }}$ is the SLD of solvent and the $b k g$ is background. The core and shell correspond to the magnetite particle and oleic acid, respectively. The $\rho_{c}, \rho_{s}$ and $\rho_{\text {solv }}$ for nuclear SLD are calculated theoretically and shown in table 4 . Fitting is carried out by using Eq.(14) with the nuclear SLDs shown in table 4. In this fitting, variable 
Table 5. Parameters to calculate $S(Q)$ for magnetic scattering.

\begin{tabular}{ll}
\hline Core radius & $r_{c}=42 \AA$ \\
\hline Shell thickness & $t=0 \AA$ \\
\hline Core SLD & $\rho_{c}=2.9 \times 10^{-10} \mathrm{~cm}^{-2}$ \\
\hline Shell SLD & $\rho_{s}=0$ \\
\hline Solvent SLD & $\rho_{\text {solv }}=0$ \\
\hline background & $b k g=0$ \\
\hline
\end{tabular}

numbers are $r_{c}, t$ and $b k g$. From this result, the core diameter is $84 \AA$ with distribution of $8.7 \%$ in full width half maximum and the shell thickness is $22 \AA$. The background $b \mathrm{~kg}$ is estimated to be 10 .

We calculate the $S(Q)$ for magnetic scattering with SLDs. The magnetic SLD for magnetite particles is calculated theoretically and used for $\rho_{c}$. Since the magnetic scattering comes only from magnetite particles, we defined $t=0, \rho_{s}=\rho_{\text {solv }}=0$ and $b k g=0$. We use the $r_{c}$ with distribution of $8.7 \%$ which is obtained by the fitting. These parameters are shown in table 5 .

The experimental data is the red circle, the fitting result with nuclear SLD is the blue dashed line and the magnetic scattering calculated with parameters shown in table 5 is the green dashed line in figure 6 . The red line in figure 6 is the sum of nuclear scattering (blue dashed line) and magnetic scattering (green dashed line), and this is consistent with the experimental data (red circle). ¿From this result, magnetic scattering is higher than nuclear scattering at $Q \simeq 0.055 \AA^{-1}$.

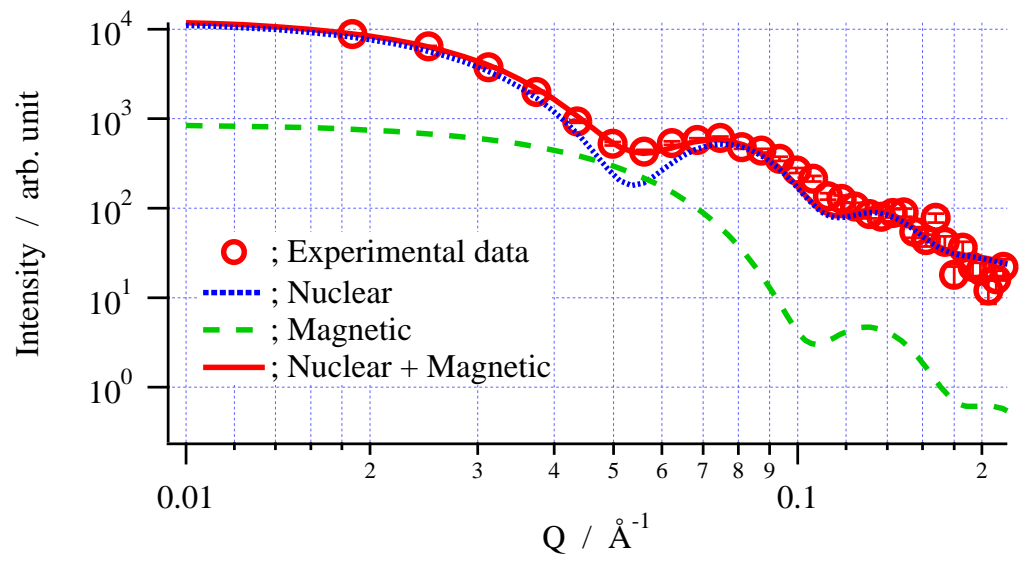

Figure 6. Red circle, blue dashed line, green dashed line and red line are experimental data, nuclear scattering, magnetic scattering and the sum of nuclear and magnetic scattering, respectively. 
Table 6. Parameters for MIEZE experiment.

\begin{tabular}{ll}
\hline$Q$ range $\left[\AA^{-1}\right]$ & $0.040,0.054,0.068,0.081,0.095,0.11$ \\
\hline$\tau$ range $[\mathrm{ns}]$ & $0.3,0.8,1.6$ \\
\hline$x_{s}[\mathrm{~mm}]$ & $4(\tau=0.3), 3(\tau=0.8), 2(\tau=1.6)$ \\
\hline$y_{s}[\mathrm{~mm}]$ & 2 \\
\hline$z_{s}[\mathrm{~mm}]$ & 20 \\
\hline$x_{d}[\mathrm{~mm}]$ & 15 \\
\hline$z_{d}[\mathrm{~mm}]$ & 55 (figure 7$), 10$ (figure 8$)$ \\
\hline
\end{tabular}

\subsection{MIEZE experiment}

We tried to measure the dynamics of magnetite ferrofluid by using the MIEZE spectrometer. The wavelength is $8.1 \AA$ and wavelength bandwidth is $17.4 \%$ in full width half maximum. Experimental parameters are shown in table 6 . The result of analysis with $z_{d}=55 \mathrm{~mm}$ is shown in figure 7 . Experimental data and MC simulation with the core shell model of $S(Q)$ are shown in dots and line, respectively. Red, blue and green correspond to $\tau=1.6,0.8,0.3 \mathrm{~ns}$, respectively. This result shows that the experimental data with large effect of path dispersion caused by the $z_{d}=55 \mathrm{~mm}$ is reproduced by $\mathrm{MC}$ simulation which is calculated with the same parameters as the experimental geometry.

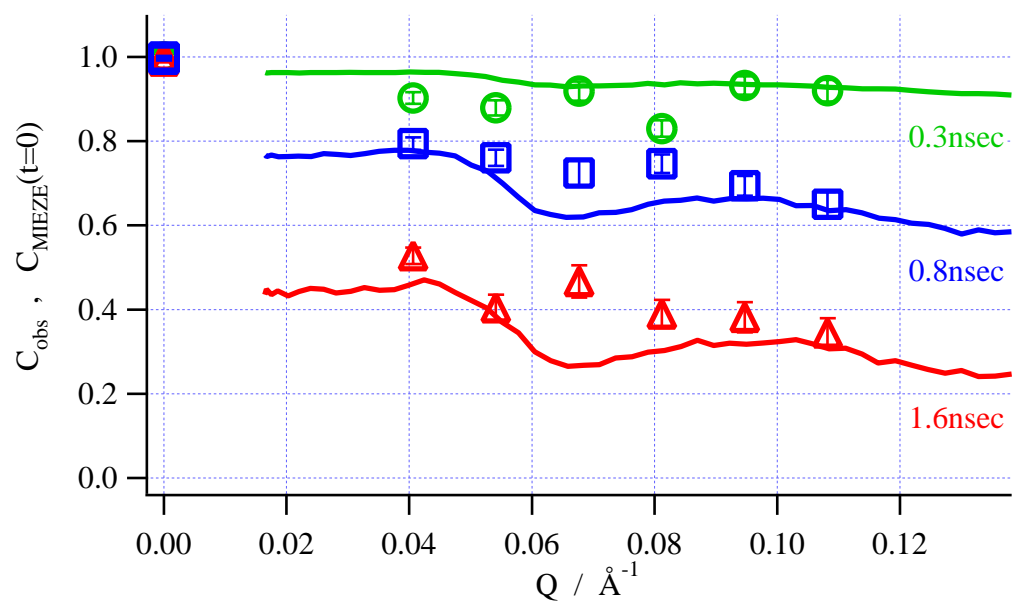

Figure 7. Red, bule, green correspond to $\tau=1.6,0.8,0.3 \mathrm{~ns}$, respectively. The experimental data and the MC simulation with $z_{d}=55 \mathrm{~mm}$ are dots and lines, respectively.

We analyzed experimental data with $z_{d}=10 \mathrm{~mm}$ and the result is shown in figure 8 . This is normalized by $C_{M I E Z E}(t=0)$ of MC simulation with $z_{d}=10 \mathrm{~mm}$. Red, blue and green 
correspond to $\tau=1.6,0.8,0.3 \mathrm{~ns}$, respectively. In the range of $Q \simeq 0.055 \AA^{-1}, C_{M I E Z E}$ decays in $\tau=1.6 \mathrm{~ns}$ comparing with other $Q$ range. In the $z_{d}=55 \mathrm{~mm}$ data, the dynamic effect is suppressed by the error because path dispersion effect is very large, but in the $z_{d}=10 \mathrm{~mm}$ data, path dispersion effects become small and dynamics effect appears. $Q \simeq 0.055 \AA^{-1}$ corresponds to the range of strong magnetic scattering as shown in figure 6. This result shows the possibility of superparamagnetism relaxation. However, the statistical accuracy is poor and we cannot yet conclude the superparamagnetism relaxation contribute to reduce the $C_{M I E Z E}$ at $Q \simeq 0.055 \AA^{-1}$. It is necessary to extract magnetic scattering only from sum of nuclear and magnetic scattering. We are planning to set an analyzer mirror after the sample position and try to observe relaxation of superparamagnetism.

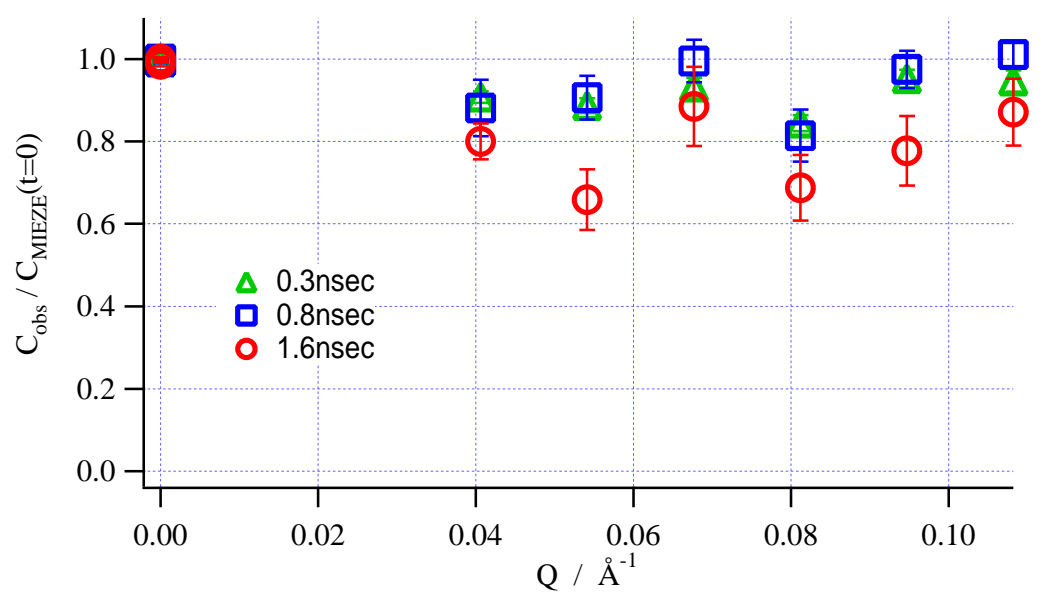

Figure 8. Red, blue, green correspond to $\tau=1.6,0.8,0.3 \mathrm{~ns}$, respectively. The experimental data with $z_{d}=10 \mathrm{~mm}$ is normalized by the MC simulation.

\section{Concluding remarks}

In the MIEZE spectrometer sample is placed after the analyzer. The contrast of MIEZE signals can be observed without reduction even in the presence of magnetic scattering which we show in this paper on a sample of magnetite ferrofluid in which average diameter is $84 \AA$. The MIEZE signal is sensitive for dispersion of path length between sample and detector. We studied the resolution function of MIEZE spectrometer by using Monte Carlo simulation. It was demonstrated that the sample thickness limit is not severe in case of small angle scattering geometry.

The experimental results were reproduced with the simulation and we estimated instrumental resolution function of the MIEZE spectrometer. It was found that $S(Q)$ for core shell model affect to the $C_{M I E Z E}(t=0)$ with wide detector size. 


\section{Acknowledgements}

The present study was supported by the Development of Systems and Technology for Advanced Measurement and Analysis (SENTAN), by JST, and through Grants-in-Aid for Scientific Research (Nos. 17760681, 17760055 and 19560841) by JSPS.

\section{References}

[1] Gähler and Golub R 1987 A high resolution neutron spectrometer for quasielastic scattering on the basis of spin-echo and magnetic resonance Z Phys. B 65269

[2] Gähler R, Golub R and Keller T, 1992 Neutron resonance spin echo-a new tool for high resolution spectroscopy Physica B 180-181 899

[3] Kawabata Y, Hino M, Kitaguchi M, Hayashida H, Tasaki S, Ebisawa T, Yamazaki D, Maruyama R, Seto H, Nagao M and Kanaya T 2006 Neutron resonance spin echo and MIEZE spectrometer development project in Japan Physica B 851122

[4] Hayashida H, Kitaguchi M, Hino M, Kawabata Y, Maruyama R and Ebisawa T 2007 Development of a resonance spin flipper for NRSE/MIEZE on a pulsed neutron beam with an oscillating frequency of $500 \mathrm{kHz}$ Nucl. Instrm. Meth. A $\mathbf{5 3 0} 292$

[5] Hayashida H, Kitaguchi M, Hino M, Kawabata Y and Ebisawa T 2007 Observation of MIEZE signal with an effective frequency of $1 \mathrm{MHz}$ Physica B 397144

[6] Bleuel M, Littrell K, Gähler R and Lal J 2005 MISANS, a method for quasi-elastic small angle neutron scattering experiments Physica B 356213

[7] Hino M, Kitaguchi M, Hayashida H, Kawabata Y, Tasaki S, Ebisawa T, Yamazaki D, Maruyama R, Tanaka K, Torikai N, Inoue R and Kanaya T 2006 A test of MIEZE-reflectometer for study of surface and interface Physica B 3851125

[8] Mezei F 1979 Lecture Notes in Physics. Neutron Spin Echo, Proceedings Grenoble 25

[9] Besenbock W, Gähler R, Hank P, Kahn R, Köppe M, De novion C, Petry W and Wuttke J 1998 First scattering experiment on MIEZE: a Fourier transform time-of-flight spectrometer using resonance coils Journal of Neutron Research $\mathbf{7} 65$

[10] Kilcoyne S H and Cywinski R 1995 Ferritin: a model superparamagnet Journal of Magnetism and Magnetic Materials 140-144 1466-1467

[11] Hirota K, Shinohara T, Ikeda K, Mishima K, Adachi T, Morishima T, Satoh S, Oku T, Yamada S, Sasao H, Suzuki J and Shimizu H.M 2005 Development of a neutron detector based on a position-sensitive photomultiplier Phys. Chem. Chem. Phys. 71836 